\title{
One-loop effects on MSSM parameter determination via chargino production at the $\mathrm{LC}$
}

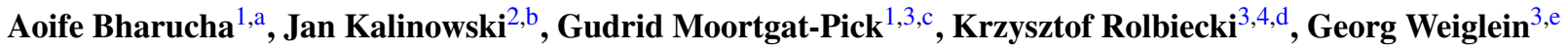 \\ ${ }^{1}$ II. Institut für Theoretische Physik, University of Hamburg, Luruper Chaussee 149, 22761 Hamburg, Germany \\ ${ }^{2}$ Faculty of Physics, University of Warsaw, 00681 Warsaw, Poland \\ ${ }^{3}$ DESY, Deutsches Elektronen-Synchrotron, Notkestr. 85, 22607 Hamburg, Germany \\ ${ }^{4}$ Instituto de Física Teórica, IFT-UAM/CSIC, 28049, Madrid, Spain
}

Received: 21 March 2013 / Published online: 8 June 2013

(C) Springer-Verlag Berlin Heidelberg and Società Italiana di Fisica 2013

\begin{abstract}
At a future linear collider very precise measurements, typically with errors of $<1 \%$, are expected to be achievable. Such an accuracy gives sensitivity to the quantum corrections, which therefore must be incorporated in theoretical calculations in order to determine the underlying new physics parameters from prospective linear collider measurements. In the context of the chargino-neutralino sector of the minimal supersymmetric standard model, this involves fitting one-loop predictions to prospective measurements of the cross sections, forward-backward asymmetries and of the accessible chargino and neutralino masses. Taking recent results from LHC SUSY and Higgs searches into account we consider three phenomenological scenarios, each with characteristic features. Our analysis shows how an accurate determination of the desired parameters is possible, providing in addition access to the stop masses and mixing angle.
\end{abstract}

\section{Introduction}

A linear collider (LC) [1-5] will be an ideal environment for high precision studies of physics beyond the standard model (BSM). A particularly well-motivated BSM theory is the minimal supersymmetric standard model (MSSM). This provides the lightest neutralino as a candidate to explain the evidence for dark matter in the universe $[6,7]$. Further, naturalness arguments (see e.g. Ref. [8]) support light higgsino-like charginos and neutralinos, as also predicted by

\footnotetext{
a e-mail: aoife.bharucha@desy.de

be-mail: jan.kalinowski@fuw.edu.pl

c e-mail: gudrid.moortgat-pick@desy.de

d e-mail: krzysztof.rolbiecki@desy.de

e e-mail: georg.weiglein@desy.de
}

GUT motivated SUSY models [9]. Due to the challenges involved in detecting electroweakinos at the LHC, current bounds coming from the ATLAS and CMS exclude only small regions of parameter space, see e.g. Refs. [10, 11]. The charginos and neutralinos could therefore be within reach of a first stage linear collider.

One approach to determine the fundamental MSSM parameters is to consider constrained models such as the constrained minimal supersymmetric standard model (CMSSM), and perform a global fit of this reduced set of parameters to all relevant experimental results available, see e.g. Ref. [12]. Here on the other hand, in order to precisely determine the nature of the underlying SUSY model, we wish to determine the fundamental parameters in the most model-independent way possible. The determination of the $\mathrm{U}(1)$ parameter $M_{1}$, the $\mathrm{SU}(2)$ parameter $M_{2}$, the higgsino parameter $\mu$ and $\tan \beta$, the ratio of the vacuum expectation values of the two neutral Higgs doublet fields, at the percent level via chargino and neutralino pair-production has been shown to be possible at LO (see Ref. [13] and references therein). Due to the expected high precision of mass and coupling measurements at the LC, as well as the fact that one-loop effects in the MSSM may be sizeable, higher order effects have to be considered. Taking these corrections into account additional MSSM parameters become relevant, such as the masses of the stops and sleptons, which are also so far weakly constrained by the LHC.

In this paper we show how it would be possible to determine the fundamental parameters of the chargino and neutralino sector at the LC, including the complications arising due to higher order effects. Specifically, we calculate the next-to-leading order (NLO) corrections to the cross-section $(\sigma)$ and forward-backward asymmetry $\left(A_{\mathrm{FB}}\right)$ for chargino production, and also to the chargino and neutralino masses. A number of next-to-leading order (NLO) calculations of 
chargino and neutralino pair production at the LC can be found in the literature [14-18]. We perform our calculations in the on-shell (OS) scheme such that, as far as possible, the mass parameters can be interpreted as the physical masses. Recent work on the OS renormalization of the charginoneutralino sector can be found in Refs. [18-24].

By fitting loop corrected predictions to these experimental results we show that it is possible to extract the fundamental parameters of the MSSM Lagrangian. However, due to the greater number of parameters, performing the fit is more involved than for the LO analysis. Choosing three potential MSSM scenarios, we assess the impact of the loop corrections and the feasibility of such an extraction in each. We further investigate the impact of obtaining masses of the charginos and neutralinos from threshold scans rather than the continuum (see Ref. [1]) on the resulting accuracy of the parameters obtained from the fit.

The paper is organized as follows. In Sect. 2 we introduce the process studied and define necessary notation. We then provide details of the calculation of the loop corrections in Sect. 3, including details of the renormalization scheme used. In Sect. 4 we further discuss the method employed in order to fit to the MSSM parameters, define the scenarios considered, and present our results. Finally in Sect. 5 we discuss the implications of the results of the fits.

\section{Process studied and tree-level relations}

In this paper we study the determination of the fundamental parameters in the chargino-neutralino sector of the MSSM, via chargino production at a LC. The charginos, $\tilde{\chi}^{ \pm}$, and neutralinos, $\tilde{\chi}^{0}$, are the mass eigenstates of the gauginos and higgsinos, as seen from the relevant part of the MSSM Lagrangian [25],

$$
\begin{aligned}
\mathcal{L}_{\tilde{\chi}}= & \overline{\tilde{\chi}_{i}^{-}}\left(\not p \delta_{i j}-P_{L}\left(U^{*} X V^{\dagger}\right)_{i j}-P_{R}\left(V X^{\dagger} U^{T}\right)_{i j}\right) \tilde{\chi}_{j}^{-} \\
& +\frac{1}{2} \overline{\tilde{\chi}_{i}^{0}}\left(\not p \delta_{i j}-P_{L}\left(N^{*} Y N^{\dagger}\right)_{i j}-P_{R}\left(N Y^{\dagger} N^{T}\right)_{i j}\right) \tilde{\chi}_{j}^{0},
\end{aligned}
$$

where $P_{L / R}=1 / 2\left(1 \mp \gamma_{5}\right)$. The mass matrix for the charginos is given by

$X=\left(\begin{array}{cc}M_{2} & \sqrt{2} M_{W} s_{\beta} \\ \sqrt{2} M_{W} c_{\beta} & \mu\end{array}\right)$,

where $s_{\beta} / c_{\beta} \equiv \sin \beta / \cos \beta$, and $M_{W}$ is the mass of the $W$ boson. This matrix is diagonalized via the bi-unitary transformation $\mathbf{M}_{\tilde{\chi}^{+}}=U^{*} X V^{\dagger}$, where $U$ and $V$ are complex unitary matrices. The mass matrix for the neutralinos in the
$\left(\tilde{B}, \tilde{W}, \tilde{H}_{1}, \tilde{H}_{2}\right)$ basis is given by

$Y=\left(\begin{array}{cccc}M_{1} & 0 & -M_{Z} c_{\beta} s_{W} & M_{Z} s_{\beta} s_{W} \\ 0 & M_{2} & M_{Z} c_{\beta} c_{W} & -M_{Z} s_{\beta} c_{W} \\ -M_{Z} c_{\beta} s_{W} & M_{Z} c_{\beta} c_{W} & 0 & -\mu \\ M_{Z} s_{\beta} s_{W} & -M_{Z} s_{\beta} c_{W} & -\mu & 0\end{array}\right)$,

where $s_{W}\left(c_{W}\right)$ is the $\sin (\cos )$ of the weak mixing angle $\theta_{W}$. Since $Y$ is complex symmetric, its diagonalization requires only one unitary matrix $N$, via $\mathbf{M}_{\tilde{\chi}^{0}}=N^{*} Y N^{\dagger}$.

As described in detail in Sect. 4, the parameter determination relies on the measurement of the masses of the charginos and neutralinos, the polarized cross-section for the pair production of charginos, $\tilde{\chi}_{1}^{-}$,

$\sigma\left(e^{+} e^{-} \rightarrow \tilde{\chi}_{1}^{+} \tilde{\chi}_{1}^{-}\right)$

and the forward-backward asymmetry defined by,

$A_{\mathrm{FB}}=\frac{\sigma(\cos \theta>0)-\sigma(\cos \theta<0)}{\sigma(\cos \theta>0)+\sigma(\cos \theta<0)}$,

for the unpolarized cross-section, where $\theta$ is the angle of the momentum of the chargino $\tilde{\chi}_{1}^{-}$with respect to the momentum of the incoming electron $e^{-}$.

Neglecting the electron-Higgs couplings, this process occurs at leading order via three diagrams, as seen in Fig. 1. The transition matrix element can be written as [26]

$$
\begin{aligned}
& \mathcal{M}_{\alpha \beta}\left(e^{+} e^{-} \rightarrow \tilde{\chi}_{i}^{+} \tilde{\chi}_{j}^{-}\right) \\
& =\frac{e}{s} Q_{\alpha \beta}\left[\bar{v}\left(e^{+}\right) \gamma_{\mu} P_{\alpha} u\left(e^{-}\right)\right]\left[\bar{u}\left(\tilde{\chi}_{j}^{-}\right) \gamma^{\mu} P_{\beta} v\left(\tilde{\chi}_{i}^{+}\right)\right],
\end{aligned}
$$

where $Q_{\alpha \beta}$ denotes the bilinear charges, $\alpha=L, R$ refers to the chirality of the $e^{+} e^{-}$current and $\beta=L, R$ to that of the $\tilde{\chi}_{i}^{+} \tilde{\chi}_{j}^{-}$current. The summation over $\alpha$ and $\beta$ is implied. The bilinear charges are comprised of the propagators and couplings

$$
\begin{aligned}
Q_{L L}= & C_{\tilde{\chi}_{i}^{+} \tilde{\chi}_{j}^{-} \gamma}^{L}-D_{Z} G_{L} C_{\tilde{\chi}_{i}^{+} \tilde{\chi}_{j}^{-} Z}^{L}, \\
Q_{R L}= & C_{\tilde{\chi}_{i}^{+} \tilde{\chi}_{j}^{-} \gamma}^{L}-D_{Z} G_{R} C_{\tilde{\chi}_{i}^{+} \tilde{\chi}_{j}^{-} Z}^{L}, \\
Q_{L R}= & C_{\tilde{\chi}_{i}^{+} \tilde{\chi}_{j}^{-} \gamma}^{R}+D_{Z} G_{L}\left(C_{\tilde{\chi}_{i}^{+} \tilde{\chi}_{j}^{-} Z}^{R}\right)^{*} \\
& +\frac{i}{2 e} D_{\tilde{v}}\left(C_{\tilde{v}_{e} e^{+} \tilde{\chi}_{i}^{-}}^{R}\right)^{*} C_{\tilde{v}_{e} e^{+} \tilde{\chi}_{j}^{-}}^{R}, \\
Q_{R R}= & C_{\tilde{\chi}_{i}^{+} \tilde{\chi}_{j}^{-} \gamma}^{R}+D_{Z} G_{R}\left(C_{\tilde{\chi}_{i}^{+} \tilde{\chi}_{j}^{-} Z}^{R}\right)^{*},
\end{aligned}
$$


Fig. 1 Tree-level diagrams for the production of charginos $\tilde{\chi}_{1}^{+}$ and $\tilde{\chi}_{1}^{-}$at the LC

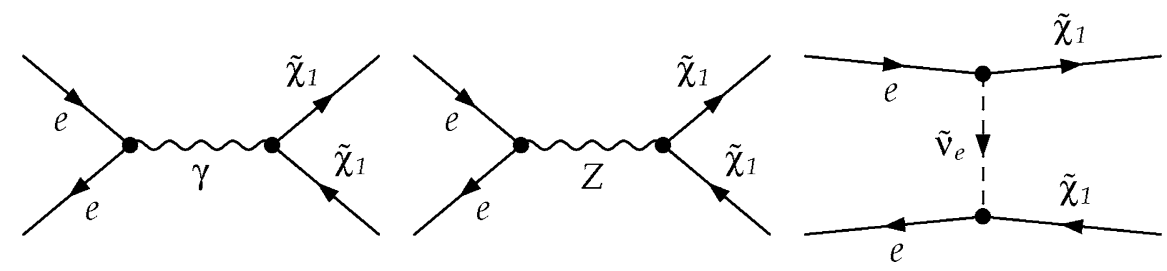

for which the required MSSM couplings for the $\tilde{\chi}_{i}^{+} \tilde{\chi}_{j}^{-} \gamma$, $\tilde{\chi}_{i}^{+} \tilde{\chi}_{j}^{-} Z$ and $e \tilde{v}_{e} \tilde{\chi}_{i}^{+}$vertices are given by

$C_{\tilde{\chi}_{i}^{+} \tilde{\chi}_{j}^{-} \gamma}^{L / R}=i e \delta_{i j}$,

$C_{\tilde{\chi}_{i}^{+} \tilde{\chi}_{j}^{-} Z}^{L}=-\frac{i e}{c_{W} s_{W}}\left(s_{W}^{2} \delta_{i j}-U_{j 1}^{*} U_{i 1}-\frac{1}{2} U_{j 2}^{*} U_{i 2}\right)$,

$C_{\tilde{x}_{i}^{+} \tilde{x}_{j}^{-} Z}^{R}=C_{\tilde{x}_{i}^{+} \tilde{x}_{j}^{-} Z}^{L}\left(U \rightarrow V^{*}\right)$,

$C_{\tilde{v}_{e} e^{+} \tilde{x}_{i}^{-}}^{R}=-\frac{i e}{s_{W}} V_{i 1}$,

and $G_{L}, G_{R}, D_{Z}$, and $D_{\tilde{v}}$ are defined via

$G_{L}=\frac{s_{W}^{2}-\frac{1}{2}}{s_{W} c_{W}}, \quad G_{R}=\frac{s_{W}}{c_{W}}$,

$D_{Z}=\frac{s}{s-M_{Z}^{2}}, \quad D_{\tilde{v}}=\frac{s}{t-m_{\tilde{\nu}}^{2}}$.

In the equations above, $e$ denotes the electric charge, $m_{e}$ and $M_{Z}$ are the masses of the electron and $Z$ boson. $D_{Z}$ and $D_{\tilde{v}}$ refer to the propagators of the $Z$ boson and sneutrino (of mass $m_{\tilde{v}}$ ), in terms of the Mandelstam variables $s$ and $t$.

One can therefore express the transition matrix element in terms of $M_{2}, \mu$ and $\tan \beta$, in addition to the known SM parameters. However, the expected accuracy of the measurements at the linear collider is such that one-loop corrections become relevant, and we shall see in the following section how the higher order expressions depend on many additional MSSM parameters.

\section{NLO contributions and renormalization}

We have calculated the full one-loop corrections to the forward-backward asymmetry for process $e^{+} e^{-} \rightarrow \tilde{\chi}_{1}^{+} \tilde{\chi}_{1}^{-}$, within the complex MSSM; the corresponding corrections to the cross section were calculated in Ref. [18]. Examples for the contributing self-energy, vertex and box diagrams are shown in Fig. 2. As in Ref. [18], for the calculation we have used the program FeynArts [27-31], which allowed an automated generation of the Feynman diagrams and amplitudes. Together with the packages FormCalc [32-34] and LoopTools [32] we derived the final matrix elements and loop integrals. We assume a unit CKM matrix. We regularize using dimensional reduction [35-37], which ensures that SUSY is preserved, via the implementation described in Refs. [32, 38].

A number of one-loop calculations in the gauginohiggsino sector can be found in the literature, mainly in the
Fig. 2 Examples of one-loop self-energy (upper), vertex (middle) and box (lower) diagrams for the production of charginos $\tilde{\chi}_{1}^{+}$and $\tilde{\chi}_{1}^{-}$at the LC (where $m, g$ denote the generation)
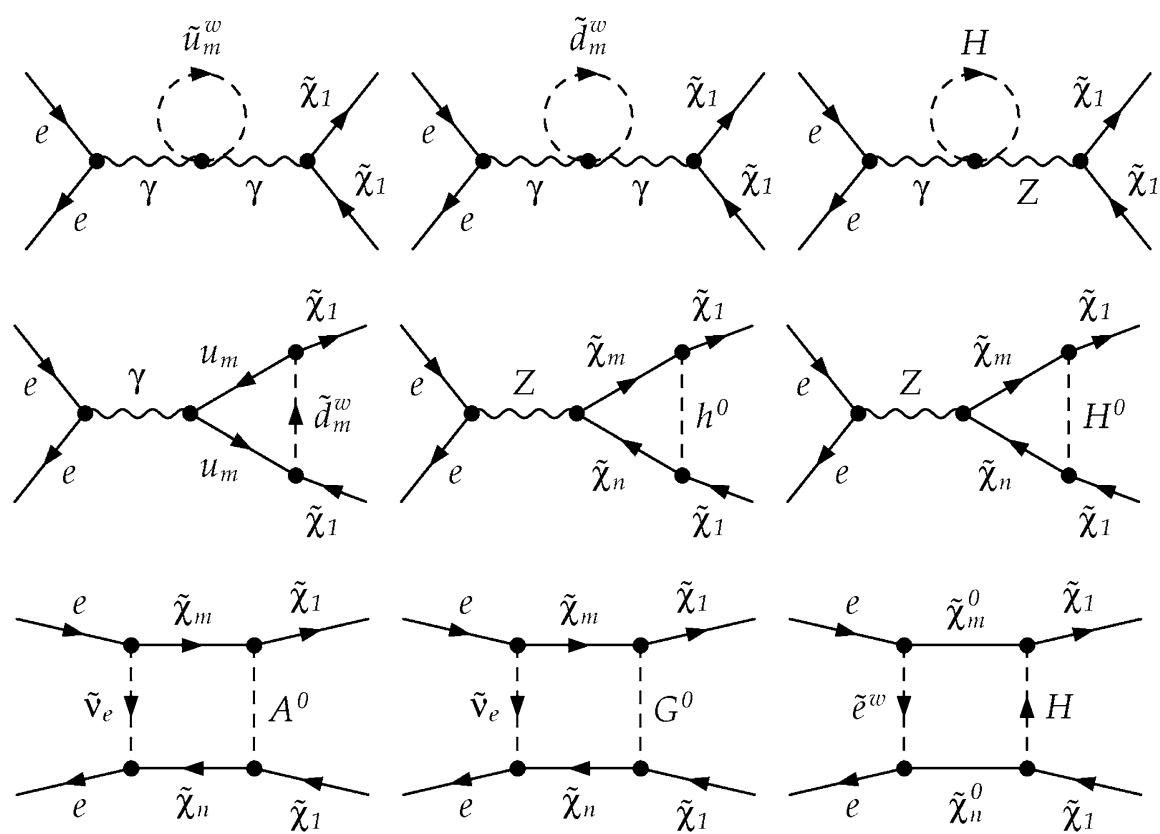
CP-conserving MSSM [14, 39-47], but some of which apply a renormalization scheme that is also applicable for complex parameters $[14,45]$. CP-odd observables have also been calculated at the one-loop level, for instance in Refs. [48-50], but no dedicated renormalization scheme was required in these cases as the observables studied were UV-finite. Since we intend to extend the current study to the case of complex parameters, here we follow the approach of Refs. [18, 20] closely, where a dedicated on-shell renormalization scheme for the chargino and neutralino sector of the MSSM with complex parameters was developed. In the following we will therefore only discuss the parameter renormalization of the chargino and neutralino sector, relevant for the definitions of the parameters at loop level, briefly and for further details about the chargino field renormalization and the renormalization of other sectors we refer the reader to Refs. [18, 20, $21,24]$.

The mass matrix in the chargino sector, Eq. (2), is renormalized via

$X \rightarrow X+\delta X$

where $\delta X$ is defined by

$\delta X=\left(\begin{array}{cc}\delta M_{2} & \sqrt{2} \delta\left(M_{W} s_{\beta}\right) \\ \sqrt{2} \delta\left(M_{W} c_{\beta}\right) & \delta \mu\end{array}\right)$,

containing the renormalization constants $\delta M_{2}$ and $\delta \mu$, as well as renormalization constants (RCs) from other sectors, $\delta c_{\beta}, \delta s_{\beta}$ (which can be expressed in terms of $\delta \tan \beta$ ), and $\delta M_{W}$, defined in Ref. [18]. The neutralino mass matrix, Eq. (3), is similarly renormalized via

$Y \rightarrow Y+\delta Y$,

where $\delta Y$ is defined analogously to $\delta X$ in Eq. (11) and contains the additional RC $\delta M_{1}$, cf. Eq. (3).

Following e.g. Ref. [20], $\delta M_{1}, \delta M_{2}$, and $\delta \mu$ are determined by choosing three out of the total six physical masses of the charginos and neutralinos to be on-shell, i.e. the treelevel masses, $m_{\tilde{\chi}_{i}}$, coincide with the one-loop renormalized masses, $M_{\tilde{\chi}_{i}}=m_{\tilde{\chi}_{i}}+\Delta m_{\tilde{\chi}_{i}}$,

$$
\begin{aligned}
\Delta m_{\tilde{\chi}_{i}} \equiv & -\frac{m_{\tilde{\chi}_{i}}}{2} \operatorname{Re}\left[\hat{\Sigma}_{i i}^{L}\left(m_{\tilde{\chi}_{i}}^{2}\right)+\hat{\Sigma}_{i i}^{R}\left(m_{\tilde{\chi}_{i}}^{2}\right)\right] \\
& -\frac{1}{2} \operatorname{Re}\left[\hat{\Sigma}_{i i}^{S L}\left(m_{\tilde{\chi}_{i}}^{2}\right)+\hat{\Sigma}_{i i}^{S R}\left(m_{\tilde{\chi}_{i}}^{2}\right)\right] \\
= & 0 .
\end{aligned}
$$

We define the coefficients $\Sigma_{i j}^{L / R}\left(p^{2}\right)$ and $\Sigma_{i j}^{S L / S R}\left(p^{2}\right)$ of the self energy via

$$
\begin{aligned}
\Sigma_{i j}\left(p^{2}\right)= & \not p P_{L} \Sigma_{i j}^{L}\left(p^{2}\right)+\not p P_{R} \Sigma_{i j}^{R}\left(p^{2}\right) \\
& +P_{L} \Sigma_{i j}^{S L}\left(p^{2}\right)+P_{R} \Sigma_{i j}^{S R}\left(p^{2}\right),
\end{aligned}
$$

and define the left and right handed vector and scalar coefficients of the renormalized self-energy analogously via $\hat{\Sigma}_{i j}^{L / R}\left(p^{2}\right)$ and $\hat{\Sigma}_{i j}^{S L / S R}\left(p^{2}\right)$, respectively.

As stated earlier, we consider the parameter renormalization as for the complex MSSM, such that our setup is easily adaptable for future extensions. In Ref. [18, 21], it was shown that in the $\mathrm{CP}$ violating case, the 1-loop corrections to the phases of $M_{1}$ and $\mu$, i.e. $\phi_{M_{1}}$ and $\phi_{\mu}$ respectively ${ }^{1}$ are UV finite. Therefore we take the approach that these phases can be left unrenormalized. We can then determine the necessary conditions to obtain the absolute values $\left|M_{1}\right|,\left|M_{2}\right|$ and $|\mu|$, depending on which three physical masses are chosen to be on-shell. As we have two external charginos, and in order to easily extend our setup to the case of $\tilde{\chi}_{1}^{+} \tilde{\chi}_{2}^{-}$production, we assume the NCC scheme with $\tilde{\chi}_{1}^{0}, \tilde{\chi}_{1}^{ \pm}$and $\tilde{\chi}_{2}^{ \pm}$ on-shell [18, 20-22], such that $i^{\prime}=1$ and $i^{\prime \prime}=1$ and 2. Note that in choosing the scheme, it is desirable that the on-shell particles should contain significant bino, wino, and higgsino components, in order that the $M_{1}, M_{2}$ and $\mu$ parameters are accessible [18, 20-22]. For the above choice, these conditions are satisfied for all the scenarios defined in Sect. 4, in which the lightest neutralino always has a sizeable binolike component. The parameters in question of the chargino mass matrix can then be renormalized via expressions given in Refs. [18, 21, 24], which we list here for completeness,

$$
\begin{aligned}
& \delta\left|M_{1}\right|=-\frac{1}{\operatorname{Re}\left(e^{-i \phi_{M_{1}}} N_{i 1}^{2}\right) F} \\
& \times\left(\left(2 \operatorname{Re}\left(e^{-i \phi_{\mu}} N_{i 3} N_{i 4}\right) \operatorname{Re}\left(U_{j 1} V_{j 1}\right)\right.\right. \\
&\left.+\operatorname{Re} N_{i 2}^{2} \operatorname{Re}\left(e^{-i \phi_{\mu}} U_{j 2} V_{j 2}\right)\right) C_{k} \\
&+\left(\operatorname{Re}\left(U_{j 1} V_{j 1}\right) \operatorname{Re}\left(e^{-i \phi_{\mu}} U_{k 2} V_{k 2}\right)\right. \\
&\left.-\operatorname{Re}\left(e^{-i \phi_{\mu}} U_{j 2} V_{j 2}\right) \operatorname{Re}\left(U_{k 1} V_{k 1}\right)\right) N_{i} \\
&-\left(\operatorname{Re} N_{i 2}^{2} \operatorname{Re}\left(e^{-i \phi_{\mu}} U_{k 2} V_{k 2}\right)\right. \\
&\left.\left.+2 \operatorname{Re}\left(e^{-i \phi_{\mu}} N_{i 3} N_{i 4}\right) \operatorname{Re}\left(U_{k 1} V_{k 1}\right)\right) C_{j}\right), \\
& \delta\left|M_{2}\right|= \frac{1}{F}\left(\operatorname{Re}\left(e^{-i \phi_{\mu}} U_{j 2} V_{j 2}\right) C_{k}\right. \\
&\left.-\operatorname{Re}\left(e^{-i \phi_{\mu}} U_{k 2} V_{k 2}\right) C_{j}\right), \\
& \delta|\mu|=- \frac{1}{F}\left(\operatorname{Re}\left(U_{j 1} V_{j 1}\right) C_{k}-\operatorname{Re}\left(U_{k 1} V_{k 1}\right) C_{j}\right) . \\
& F, C_{i}, \text { and } N_{i} \text { are defined by }
\end{aligned}
$$

$$
\begin{aligned}
F= & 2\left(\operatorname{Re}\left(U_{k 1} V_{k 1}\right) \operatorname{Re}\left(e^{-i \phi_{\mu}} U_{j 2} V_{j 2}\right)\right. \\
& \left.-\operatorname{Re}\left(U_{j 1} V_{j 1}\right) \operatorname{Re}\left(e^{-i \phi_{\mu}} U_{k 2} V_{k 2}\right)\right),
\end{aligned}
$$

\footnotetext{
${ }^{1}$ We adopt the convention that $M_{2}$ is real.
} 


$$
\begin{aligned}
C_{i}= & \operatorname{Re}\left[m _ { \tilde { \chi } _ { i } ^ { + } } \left[\Sigma_{ \pm, i i}^{L}\left(m_{\tilde{\chi}_{i}^{+}}^{2}\right)\right.\right. \\
& \left.\left.+\Sigma_{ \pm, i i}^{R}\left(m_{\tilde{\chi}_{i}^{+}}^{2}\right)\right]+\Sigma_{ \pm, i i}^{S L}\left(m_{\tilde{\chi}_{i}^{+}}^{2}\right)+\Sigma_{ \pm, i i}^{S R}\left(m_{\tilde{\chi}_{i}^{+}}^{2}\right)\right] \\
& -\sum_{\substack{j=1,2 \\
k=1,2}} 2 \delta X_{j k} \operatorname{Re}\left(U_{i j} V_{i k}\right), \\
N_{i}= & \operatorname{Re}\left[m _ { \tilde { \chi } _ { i } ^ { 0 } } \left[\Sigma_{0, i i}^{L}\left(m_{\tilde{\chi}_{i}^{0}}^{2}\right)\right.\right. \\
& \left.\left.+\Sigma_{0, i i}^{R}\left(m_{\tilde{\chi}_{i}^{0}}^{2}\right)\right]+\Sigma_{0, i i}^{S L}\left(m_{\tilde{\chi}_{i}^{0}}^{2}\right)+\Sigma_{0, i i}^{S R}\left(m_{\tilde{\chi}_{i}^{0}}^{2}\right)\right] \\
& -\sum_{\substack{j=1,2 \\
k=3,4}} 4 \delta Y_{j k} \operatorname{Re}\left(N_{i j} N_{i k}\right),
\end{aligned}
$$

and the subscripts \pm and 0 identify the coefficients of the chargino and neutralino self-energy, respectively. ${ }^{2}$

Finite results for the process of interest at one-loop are obtained by adding the counterterm diagrams shown in Fig. 3. Although FeynArts generates these diagrams, expressions for the counterterms which renormalize the couplings defined at tree-level in Eq. (6), calculated in Ref. [18], are required as input, and therefore, again for completeness, we provide expressions for these explicitly. For the $\gamma \tilde{\chi}_{i}^{+} \tilde{\chi}_{j}^{-}$, $Z \tilde{\chi}_{i}^{+} \tilde{\chi}_{j}^{-}$and $e \tilde{v}_{e} \tilde{\chi}_{i}^{+}$vertices, these can be expressed as follows:

$$
\begin{aligned}
\delta C_{\tilde{x}_{i}^{+} \tilde{\chi}_{j}^{-} \gamma}^{L}= & C_{\tilde{\chi}_{i}^{+} \tilde{\chi}_{j}^{-} \gamma}^{L}\left(\delta Z_{e}+\frac{\delta Z_{\gamma \gamma}}{2}\right) \\
& +C_{\tilde{\chi}_{i}^{+} \tilde{\chi}_{j}^{-} Z}^{L} \frac{\delta Z_{Z \gamma}}{2}+\frac{i e}{2}\left(\delta Z_{ \pm, i j}^{L}+\delta \bar{Z}_{ \pm, i j}^{L}\right), \\
\delta C_{\tilde{\chi}_{i}^{+} \tilde{\chi}_{j}^{-} Z}^{L}= & C_{\tilde{\chi}_{i}^{+} \tilde{\chi}_{j}^{-} Z}^{L}\left(\delta Z_{e}-\frac{\delta c_{W}}{c_{W}}-\frac{\delta s_{W}}{s_{W}}+\frac{\delta Z_{Z Z}}{2}\right) \\
& +C_{\tilde{\chi}_{i}^{+} \tilde{\chi}_{j}^{-} \gamma}^{L} \frac{\delta Z_{\gamma Z}}{2}-2 i e \frac{\delta s_{W}}{c_{W}} \delta_{i j} \\
& +\frac{1}{2} \sum_{n=1,2}\left(\delta C_{\tilde{\chi}_{i}^{+}}^{L} \tilde{\chi}_{n}^{-} Z^{Z_{ \pm, n j}}+C_{\tilde{\chi}_{n}^{+} \tilde{\chi}_{j}^{-} Z^{\delta}}^{L} \bar{Z}_{ \pm, i n}^{L}\right),
\end{aligned}
$$

${ }^{2}$ Here $N_{i}$ should not be confused with the neutralino mass matrix $N_{i j}$. where the analogous right-handed parts are obtained by the replacement $L \rightarrow R$, and

$$
\begin{aligned}
\delta C_{\tilde{v}_{e} e^{+} \tilde{\chi}_{i}^{-}}^{R}= & C_{\tilde{v}_{e} e^{+}}^{R} \tilde{\chi}_{i}^{-}\left(\delta Z_{e}-\frac{\delta s_{W}}{s_{W}}+\frac{1}{2}\left(\delta Z_{\tilde{v}_{e}}+\delta Z_{e}^{L^{*}}\right)\right) \\
& +\frac{1}{2}\left(C_{\tilde{v}_{e} e^{+} \tilde{\chi}_{1}^{-}}^{R} \delta Z_{ \pm, 1 i}^{R}+C_{\tilde{v}_{e} e^{+} \tilde{\chi}_{2}^{-}}^{R} \delta Z_{ \pm, 2 i}^{R}\right) .
\end{aligned}
$$

Note that the renormalization constants of the SM fields, i.e. $Z_{V V}(V=\gamma, Z)$ and $\delta Z_{e}^{L}$ for the vector bosons and electron, and parameters, i.e. $\delta Z_{e}$ and $\delta c_{W}\left(s_{W}\right)$ for the electric charge and $\cos (\sin )$ of the weak mixing angle respectively, can be found in Ref. [18]. The renormalization for the chargino fields is performed in the most general manner, making use of separate RCs for the incoming and outgoing fields, i.e. coefficients $\delta Z_{ \pm, i j}^{L / R}$ and $\delta \bar{Z}_{ \pm, i j}^{L / R}$, respectively, for left- and right-handed charginos as given in Ref. [18]. Finally, the counterterm for the sneutrino self energy takes the form

$$
\begin{aligned}
\delta C_{\tilde{\tilde{v}}_{i} \tilde{v}_{j}}= & i \delta_{i j}\left(\frac{1}{2}\left(\delta Z_{\tilde{v}_{i}}+\delta Z_{\tilde{v}_{i}}^{*}\right) p^{2}-\delta m_{\tilde{v}_{i}}^{2}\right. \\
& \left.-\frac{m_{\tilde{v}_{i}}^{2}}{2}\left(\delta Z_{\tilde{v}_{i}}+\delta Z_{\tilde{v}_{i}}^{*}\right)\right),
\end{aligned}
$$

for $\tilde{v}_{i}=\tilde{v}_{e}, \tilde{v}_{\mu}, \tilde{v}_{\tau}$, where the sneutrino field and mass RCs, $\delta Z_{\tilde{v}_{i}}^{*}$ and $\delta m_{\tilde{v}_{i}}$, are also defined following Ref. [18].

Initial and final state soft radiation must also be included to obtain an infra-red finite result as the incoming and outgoing particles are charged, and this is done as described in detail in Ref. [18], using the phase-space slicing method to define the singular soft and collinear contributions in the regions $E<\Delta E$ and $\theta<\Delta \theta$, respectively. In the soft and collinear limit, the results are regularized using electron and photon masses, respectively, and factorized into analytically integrable expressions proportional to the tree-level crosssection $\sigma^{\text {tree }}\left(e^{+} e^{-} \rightarrow \tilde{\chi}_{1}^{+} \tilde{\chi}_{1}^{-}\right)$. However, the result is cut-off dependent (i.e. on $\Delta E$ and $\Delta \theta$ ), and removing this dependence requires a calculation of the cross section for the three body final state, excluding the soft and collinear regions, which we perform using FeynArts and Formcalc. We further require that soft photon radiation is included in the
Fig. 3 Counterterm diagrams in the MSSM for the production of charginos $\tilde{\chi}_{1}^{+}$and $\tilde{\chi}_{1}^{-}$at the LC

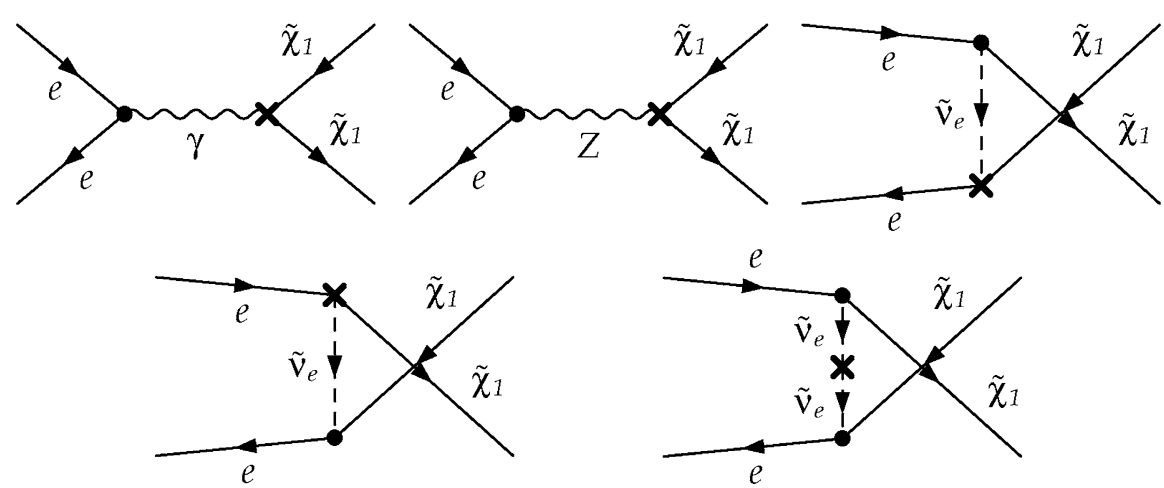


cross-section obtained from FormCalc. Finally we obtain a complete IR finite and cut-off independent result by adding the collinear contribution, which is calculated following the procedure outlined in Ref. [14].

\section{Fit strategy and numerical results}

\subsection{Obtaining MSSM parameters from the fit}

With the loop corrections calculated as in Sect. 3, we can determine the fundamental parameters of the MSSM at NLO. From now on, we will restrict our study to the case of real parameters. In the chargino and neutralino sectors there are four real parameters, see Sect. 2, which we fit to,

$M_{1}, \quad M_{2}, \quad \mu, \quad \tan \beta$.

We additionally fit to the sneutrino mass, as this enters at tree level and will therefore significantly affect cross sections and forward-backward asymmetries. However, in those scenarios where the sneutrino would already have been observed at the LC, its mass is assumed to be known. At the loop level, a large number of MSSM parameters will contribute. Depending on the scenario, only limited knowledge about some of these may be available. In particular LHC data may only provide limited information about the parameters of the stop sector, and direct production at the LC might not be possible. However, our analysis also offers good sensitivity to these parameters at the LC, as stops could significantly contribute to chargino/neutralino observables at NLO.

At the LC, masses are expected to be measured with high precision using different methods [1]. In the following we adopt the experimental precision which could be achieved using the threshold scan method, however, we also investigate how the fit precision would change if the masses were obtained from the continuum. In case of the cross sections, the experimental uncertainty is dominated by the statistical uncertainty [51],

$$
\frac{\Delta \sigma}{\sigma}=\frac{\sqrt{S+B}}{S},
$$

where $S$ and $B$ are the signal and background contributions, respectively. In addition, we assume that the statistical uncertainties for the cross sections correspond to an integrated luminosity of $\mathcal{L}=200 \mathrm{fb}^{-1}$ per polarization assuming the efficiency of $\epsilon=15 \%$, which includes branching ratios for semileptonic final states and a selection efficiency of $50 \%$ [51]. Similarly, for the forward-backward asymmetry we have

$\delta A_{\mathrm{FB}}^{\mathrm{stat}}=\sqrt{\frac{1-A_{\mathrm{FB}}^{2}}{N}}$,

and the total number of events $N=N_{+}+N_{-}$[51].
In order to estimate the theoretical uncertainty on the masses, cross-sections and forward-backward asymmetries, we consider the size of possible effects due to neglected higher order corrections as well as unknown MSSM parameters not included in the fit. NNLO corrections are an important source of theoretical uncertainty, however, at present, corrections of this kind are only known for chargino and neutralino masses, for which the leading SUSY-QCD NNLO corrections were calculated in Ref. [46, 47]. Based on these results we estimate the uncertainty on the masses due to NNLO corrections to be of the order of $0.5 \mathrm{GeV}$, i.e. comparable to the expected experimental uncertainty. Note that the masses chosen on-shell are assigned no theoretical uncertainty. We further neglect the currently unknown uncertainties arising due to NNLO corrections to the cross-sections and forward-backward asymmetries, assuming that in the future NNLO results for these could be incorporated. However, we do include the additional uncertainty arising due to any unknown MSSM parameters which are not included in the fit, dominated by the contribution from the heavy pseudoscalar Higgs boson mass $M_{A^{0}}$. We perform a multi-dimensional $\chi^{2}$ fit using Minuit $[52,53]$

$\chi^{2}=\sum_{i}\left|\frac{\mathcal{O}_{i}-\overline{\mathcal{O}}_{i}}{\delta \mathcal{O}_{i}}\right|^{2}$,

where the sum runs over the input observables $\mathcal{O}_{i}$, depending on the scenario, with their corresponding experimental uncertainties $\delta \mathcal{O}_{i}$.

\subsection{Scenarios studied and motivation}

We carry out the fit for three scenarios, S1, S2 and S3, shown in Table 1, chosen in order to realistically assess the sensitivity to the desired parameters in a number of possible situations. Due to the current status of direct LHC searches [54, 55], in all scenarios we require heavy first and second generation squarks and gluinos, while the stop sector is assumed to be relatively light. ${ }^{3}$ In S1 and S2 we take the masses of the stops, $m_{\tilde{t}_{1}}$ and $m_{\tilde{t}_{2}}$, to be $400 \mathrm{GeV}$ and $800 \mathrm{GeV}$ respectively, and the mixing angle to be $\cos \theta_{t}=0$. The sbottom sector can then be obtained by defining $m_{\tilde{b}_{1}}=$ $400 \mathrm{GeV}$ and $\cos \theta_{b}=0$. On the other hand in S3, in order to obtain $m_{h}=125 \mathrm{GeV}$, calculated using FeynHiggs 2.9.1 [56-59], such that it is compatible with the recent Higgs results from the LHC $[60,61]$, the stop sector parameters are chosen to be $m_{u_{3}}=450 \mathrm{GeV}, m_{q_{3}}=1500 \mathrm{GeV}$ and $A_{t}=-1850 \mathrm{GeV}$, ensuring large mixing between the stops,

\footnotetext{
${ }^{3}$ Note that in light of current LHC limits, the value $M_{3}=700 \mathrm{GeV}$ in S1 and S2 means that the gluino mass is rather low, however, our results are largely independent of this choice as $M_{3}$ only enters our calculations via two loop corrections to $m_{h}$.
} 
Table 1 Parameters for scenarios $1 / 2$ and 3 (S1/S2 and S3), in GeV with the exception of $\tan \beta$. Here $M_{(l / q)_{i}}\left(M_{(e / u / d)_{i}}\right)$ represents the left (right) handed mass parameter for a slepton/squark of generation $i$ respectively (jointly referred to as $M_{f_{i}}$ ), and $A_{f}$ is the trilinear coupling for a sfermion $\tilde{f}$. See text for stop and sbottom parameter definitions

Scenario $1 / 2$

\begin{tabular}{lrlr}
\hline$M_{1}$ & 125 & $M_{2}$ & $250 / 2000$ \\
$\mu$ & 180 & $M_{A^{0}}$ & 1000 \\
$M_{3}$ & 700 & $\tan \beta$ & 10 \\
$M_{q_{1,2}}$ & 1500 & $A_{q_{1,2}}$ & 650 \\
$M_{l / e_{1,2}}$ & 1500 & $A_{l_{i}}$ & 650 \\
$M_{l_{3}}$ & 800 & $M_{e_{3}}$ & 400 \\
\hline
\end{tabular}

Scenario 3

\begin{tabular}{lrlr}
\hline$M_{1}$ & 106 & $M_{2}$ & 212 \\
$\mu$ & 180 & $M_{A^{0}}$ & 500 \\
$M_{3}$ & 1500 & $\tan \beta$ & 12 \\
$M_{q_{1,2}}$ & 1500 & $A_{q_{1,2}}$ & -1850 \\
$M_{l_{i}}$ & 180 & $A_{l_{i}}$ & -1850 \\
$M_{e_{1,2}}$ & 125 & $M_{e_{3}}$ & 106 \\
\hline
\end{tabular}

such that $\cos \theta_{t}=0.148$. The sbottom sector is then obtained by defining $m_{\tilde{b}_{1}}=450 \mathrm{GeV}$ and $\cos \theta_{b}=0$. In Fig. 4 , for each of these scenarios, the mass corrections for neutralinos $\tilde{\chi}_{2}^{0}$ and $\tilde{\chi}_{3}^{0}$ are seen to be sensitive to the stop mixing angle.
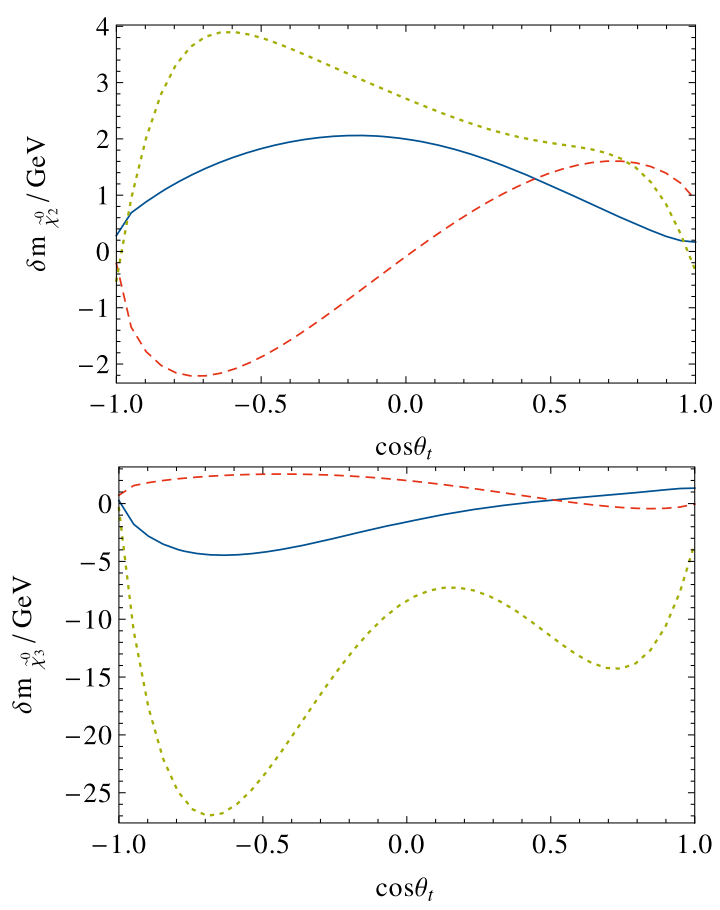

Fig. 4 One-loop corrections to the masses of neutralinos $\tilde{\chi}_{2}^{0}$ (upper) and $\tilde{\chi}_{3}^{0}$ (lower) as a function of the stop mixing angle $\cos \theta_{t}$, for scenarios S1 (blue), S2 (red, dashed) and S3 (green, dotted)
As a result of indirect limits (checked using micrOmegas 2 .4.1[62,63]), we have chosen mixed gauginohiggsino scenarios favored by the relic density measurements [64] and relatively high pseudoscalar Higgs masses in light of flavor physics constraints, e.g. the branching ratio of $\mathcal{B}\left(B_{s} \rightarrow \mu^{+} \mu^{-}\right)$[65]. We also check that our scenarios agree with the experimental results for branching ratio $\mathcal{B}(b \rightarrow s \gamma)$ and the anomalous magnetic moment of the muon $\Delta\left(g_{\mu}-2\right) / 2$. Further, in S2 we study the sensitivity of the fit to large values of $M_{2}$, such that the wino-like chargino and neutralino are heavy and decoupled from the bino- and higgsino-like particles. Finally, in S1/S2 we consider the case that the sleptons (with the exception of the light stau) and pseudoscalar Higgs bosons are at the $\mathrm{TeV}$ scale, and in S3 the case that they are relatively light. Therefore, while S1/S2 are not in keeping with the $125 \mathrm{GeV}$ Higgs boson, they provide illustrative examples of the potential of the LC in scenarios complementary to $\mathrm{S} 3 .^{4}$

\subsection{Results for scenario 1}

In this scenario, only the charginos and three neutralinos will be accessible at the LC. As input for the fit we therefore use:

- the masses of the charginos $\left(\tilde{\chi}_{1}^{ \pm}, \tilde{\chi}_{2}^{ \pm}\right)$and three lightest neutralinos $\left(\tilde{\chi}_{1}^{0}, \tilde{\chi}_{2}^{0}, \tilde{\chi}_{3}^{0}\right)$

- the light chargino production cross section $\sigma\left(\tilde{\chi}_{1}^{+} \tilde{\chi}_{1}^{-}\right)$ with polarized beams at $\sqrt{s}=350$ and $500 \mathrm{GeV}$

- the forward-backward asymmetry $A_{\mathrm{FB}}$ at $\sqrt{s}=350$ and $500 \mathrm{GeV}$

- the branching ratio $\mathcal{B}(b \rightarrow s \gamma)$ calculated using micrOmegas 2.4.1[62,63].

The input variables, together with errors, namely the assumed experimental precision of the prospective LC measurements as well as the theoretical uncertainties, are listed in Table 2. It is interesting to observe the large NLO corrections to $A_{\mathrm{FB}}$, which even result in a change of sign. Note that $\mathcal{B}(b \rightarrow s \gamma)$ is included in order to increase sensitivity to the third generation squark sector, and the estimated experimental precision of $0.3 \cdot 10^{-4}$, taken from Ref. [66], is adopted. We found that the impact of the muon anomalous magnetic moment is negligible in this scenario, mainly due to the heavy smuon sector. It should be possible to probe the supersymmetric QCD sector, with squark masses of $\sim 1.5 \mathrm{TeV}$ and the gluino mass of $\sim 700 \mathrm{GeV}$, at the LHC, such that the theoretical uncertainty arising due to these parameters is small in comparison to that due to the unknown $M_{A^{0}}$. We therefore include the small dependence on the $A^{0}$ mass as an additional source of error, having explicitly checked that the impact of all other parameters is negligible. Note that there

\footnotetext{
${ }^{4}$ Note that in S1(S2) a Higgs mass of $m_{h}=125 \mathrm{GeV}$ can also be achieved by adopting $\cos \theta_{t}=-0.4(-0.5)$.
} 
Table 2 Observables (masses in $\mathrm{GeV}$, cross sections in $\mathrm{fb}$ ) used as input for the fit in S1, tree-level values and loop corrections are specified. Here the superscript on $\sigma$ and $A_{\mathrm{FB}}$ denotes $\sqrt{s}$ in $\mathrm{GeV}$, and the subscript on $\sigma$ denotes the beam polarization $\left(\mathcal{P}\left(e^{-}\right), \mathcal{P}\left(e^{+}\right)\right)$

The central value of the theoretical prediction,

$\mathcal{B}(b \rightarrow s \gamma)=3.3 \cdot 10^{-4} \mathrm{GeV}$, calculated using state-of-the-art tools, is also included in the fit. Errors in brackets are for masses obtained from the continuum. See text for details of error estimation

\begin{tabular}{lccll}
\hline Observable & Tree value & Loop corr. & Error exp. & Error th. \\
\hline$m_{\tilde{\chi}_{1}^{ \pm}}$ & 149.6 & - & $0.1(0.2)$ & - \\
$m_{\tilde{\chi}_{2}^{ \pm}}$ & 292.3 & - & $0.5(2.0)$ & - \\
$m_{\tilde{\chi}_{1}^{0}}$ & 106.9 & - & 0.2 & - \\
$m_{\tilde{\chi}_{2}^{0}}$ & 164.0 & 2.0 & $0.5(1.0)$ & 0.5 \\
$m_{\tilde{\chi}_{3}^{0}}$ & 188.6 & -1.5 & $0.5(1.0)$ & 0.5 \\
$\sigma\left(\tilde{\chi}_{1}^{+} \tilde{\chi}_{1}^{-}\right)_{(-0.8,0.6)}^{350}$ & 2347.5 & -291.3 & 8.7 & 2.0 \\
$\sigma\left(\tilde{\chi}_{1}^{+} \tilde{\chi}_{1}^{-}\right)_{(0.8,-0.6)}^{350}$ & 224.4 & 7.6 & 2.7 & 0.5 \\
$\sigma\left(\tilde{\chi}_{1}^{+} \tilde{\chi}_{1}^{-}\right)_{(-0.8,0.6)}^{500}$ & 1450.6 & -24.4 & 8.7 & 2.0 \\
$\sigma\left(\tilde{\chi}_{1}^{+} \tilde{\chi}_{1}^{-}\right)_{(0.8,-0.6)}^{500}$ & 154.8 & 12.7 & 2.0 & 0.5 \\
$A_{\mathrm{FB}}^{350}(\%)$ & -2.2 & 6.8 & 0.8 & 0.1 \\
$A_{\mathrm{FB}}^{500}(\%)$ & -2.6 & 5.3 & 1.0 & 0.1 \\
\hline
\end{tabular}

are no theoretical errors for masses chosen to be on-shell. Even at one loop, these masses are related to the fundamental parameters via the tree level relations, and are included in the fit.

In S1 we fit eight MSSM parameters: $M_{1}, M_{2}, \mu, \tan \beta$, $m_{\tilde{v}}, \cos \theta_{t}, m_{\tilde{t}_{1}}$, and $m_{\tilde{t}_{2}}$. The results of the fit are given in Table 3. We find that the gaugino and higgsino mass parameters are determined with an accuracy better than $1 \%$, while $\tan \beta$ is determined with an accuracy of $5 \%$. Excellent precision of $2-3 \%$ is obtained for the mass of the otherwise unobservable sneutrino. Including NLO effects even allows us to constrain the parameters of the stop sector. Although the precision shown in Table 3 is rather limited, this could lead to an important hint concerning the masses of the stops, which, if not already seen, might allow for a well-targeted search at the LHC. This could be another example of LCLHC interplay [67].

Finally, in Table 3 we compare the fit results using masses of the charginos and neutralinos obtained from threshold scans and from the continuum. For the latter, the accuracy at which the parameters can be determined is seen to de-

Table 3 Fit results (masses in $\mathrm{GeV}$ ) for S1, for masses obtained from threshold scans (threshold fit) and from the continuum (continuum fit). Numbers in brackets denote $2 \sigma$ errors

\begin{tabular}{lll}
\hline Parameter & Threshold fit & Continuum fit \\
\hline$M_{1}$ & $125 \pm 0.3( \pm 0.7)$ & $125 \pm 0.6( \pm 1.2)$ \\
$M_{2}$ & $250 \pm 0.6( \pm 1.3)$ & $250 \pm 1.6( \pm 3)$ \\
$\mu$ & $180 \pm 0.4( \pm 0.8)$ & $180 \pm 0.7( \pm 1.3)$ \\
$\tan \beta$ & $10 \pm 0.5( \pm 1)$ & $10 \pm 1.3( \pm 2.6)$ \\
$m_{\tilde{v}}$ & $1500 \pm 24\left({ }_{-40}^{+60}\right)$ & $1500 \pm 20( \pm 40)$ \\
$\cos \theta_{t}$ & $0 \pm 0.15\left({ }_{-0.3}^{+0.4}\right)$ & - \\
$m_{\tilde{t}_{1}}$ & $400_{-120}^{+180}\left({ }_{\text {at limit lit }}^{\text {at limit }}\right)$ & - \\
$m_{\tilde{t}_{2}}$ & $800_{-170}^{+300}\left({ }_{-290}^{+1000}\right)$ & $800_{-220}^{+350}\left(\begin{array}{c}\text { at limit limit } \\
\text { at lim }\end{array}\right)$ \\
\hline
\end{tabular}

teriorate, with errors on the fundamental parameters almost doubling, clearly indicating the need to measure chargino and neutralino masses via threshold scans.

\subsection{Results for scenario 2}

In this scenario, where the $M_{2}$ parameter is set to $2 \mathrm{TeV}$, only the light chargino and three lightest neutralinos will be accessible at the LC. As input for the fit we therefore use:

- the masses of the lighter chargino $\left(\tilde{\chi}_{1}^{ \pm}\right)$and neutralinos $\left(\tilde{\chi}_{1}^{0}, \tilde{\chi}_{2}^{0}, \tilde{\chi}_{3}^{0}\right)$

- the light chargino production cross section $\sigma\left(\tilde{\chi}_{1}^{+} \tilde{\chi}_{1}^{-}\right)$ with polarized beams at $\sqrt{s}=400$ and $500 \mathrm{GeV}$

- the forward-backward asymmetry $A_{\mathrm{FB}}$ at $\sqrt{s}=400$ and $500 \mathrm{GeV}$

- the branching ratio $\mathcal{B}(b \rightarrow s \gamma)$.

As we again find that the muon anomalous magnetic moment has a negligible impact, it is not used in the fit. The input variables, together with errors, namely the assumed experimental precision of the prospective LC measurements as well as the theoretical uncertainties, are listed in Table 4. While $A_{\mathrm{FB}}$ is negligible at LO, the NLO corrections to it are again found to be large.

We again fit eight MSSM parameters: $M_{1}, M_{2}, \mu, \tan \beta$, $m_{\tilde{v}}, \cos \theta_{t}, m_{\tilde{t}_{1}}$, and $m_{\tilde{t}_{2}}$. The impact of other parameters, except the heavy Higgs boson mass, can be neglected. The results from the fit are given in Table 5. The higgsino and bino mass parameters are well constrained in this scenario since bino-like neutralino and all higgsinos are directly accessible. Even though the winos are not directly accessible, the wino mass parameter $M_{2}$ can be constrained with $10 \%$ accuracy at $1 \sigma$ level. An accuracy of $20 \%$ is achieved for $\tan \beta$, significantly worse than in $\mathrm{S} 1$. This can be understood by the fact that the mixing in $\mathrm{S} 2$ between chargino states is weak due to $M_{2}$ being heavy, and the constraint on $\tan \beta$ is 
Table 4 Observables (masses in $\mathrm{GeV}$, cross sections in $\mathrm{fb}$ ) used as an input for the fit in S2, as in Table 4. The central value of the theoretical prediction, $\mathcal{B}(b \rightarrow s \gamma)=3.3 \cdot 10^{-4} \mathrm{GeV}$, calculated using state-of-the-art tools, is also included in the fit. See text for details of error estimation

\begin{tabular}{lccll}
\hline Observable & Tree value & Loop corr. & Error exp. & Error th. \\
\hline$m_{\tilde{\chi}_{1}^{ \pm}}$ & 179.1 & - & 0.1 & - \\
$m_{\tilde{\chi}_{1}^{0}}$ & 111.1 & - & 0.2 & - \\
$m_{\tilde{\chi}_{2}^{0}}$ & 183.6 & 0.07 & 0.5 & 0.5 \\
$m_{\tilde{\chi}_{3}^{0}}$ & 194.2 & 1.9 & 0.5 & 0.5 \\
$\sigma\left(\tilde{\chi}_{1}^{+} \tilde{\chi}_{1}^{-}\right)_{(-0.8,0.6)}^{400}$ & 1214.9 & -344.7 & 6.0 & 0.1 \\
$\sigma\left(\tilde{\chi}_{1}^{+} \tilde{\chi}_{1}^{-}\right)_{(0.8,-0.6)}^{400}$ & 250.6 & -32.4 & 2.7 & 0.1 \\
$\sigma\left(\tilde{\chi}_{1}^{+} \tilde{\chi}_{1}^{-}\right)_{(-0.8,0.6)}^{500}$ & 1079.2 & -194.8 & 6.0 & 0.1 \\
$\sigma\left(\tilde{\chi}_{1}^{+} \tilde{\chi}_{1}^{-}\right)_{(0.8,-0.6)}^{500}$ & 229.6 & -8.7 & 2.7 & 0.1 \\
$A_{\mathrm{FB}}^{400}(\%)$ & 0.0 & 3.0 & 1.0 & 0.1 \\
$A_{\mathrm{FB}}^{500}(\%)$ & 0.0 & 5.0 & 1.0 & 0.1 \\
\hline
\end{tabular}

Table 5 Fit results (in $\mathrm{GeV}$ with the exception of $\tan \beta$ and $\cos \theta_{t}$ ) for $\mathbf{S} 2$, as in Table 3, where numbers in brackets denote $2 \sigma$ errors

\begin{tabular}{ll}
\hline Parameter & Fit result \\
\hline$M_{1}$ & $125_{-0.6}^{+0.9}\left({ }_{-1.2}^{+2.1}\right)$ \\
$M_{2}$ & $2000 \pm 200\left({ }_{-400}^{+600}\right)$ \\
$\mu$ & $180 \pm 0.2\left({ }_{-0.3}^{+0.5}\right)$ \\
$\tan \beta$ & $10 \pm 2\left({ }_{-4}^{+5}\right)$ \\
$m_{\tilde{\nu}}$ & unconstrained \\
$\cos \theta_{t}$ & $0_{-0.09}^{+0.13}\left({ }_{-0.3}^{+0.4}\right)$ \\
$m_{\tilde{t}_{1}}$ & $400_{-50}^{+250}\left({ }_{-80}^{+500}\right)$ \\
$m_{\tilde{t}_{2}}$ & $800_{-200}^{+300}\left({ }_{-400}^{+900}\right)$ \\
\hline
\end{tabular}

dependent on this mixing. No limits can be derived on the sneutrino mass, due to the Yukawa suppressed coupling of the higgsino-like $\tilde{\chi}_{1}^{ \pm}$to the electron and sneutrino. We are, however, as shown in Table 5, still able to derive limits on the stop masses and mixing parameter.

\subsection{Results for parameters in scenario 3}

This final scenario features the richest phenomenology of the studied benchmark scenarios. As input for the fit we therefore use:

- the masses of the charginos $\left(\tilde{\chi}_{1}^{ \pm}, \tilde{\chi}_{2}^{ \pm}\right)$and neutralinos $\left(\tilde{\chi}_{1}^{0}, \tilde{\chi}_{2}^{0}, \tilde{\chi}_{3}^{0}\right)$

- the light chargino production cross section $\sigma\left(\tilde{\chi}_{1}^{+} \tilde{\chi}_{1}^{-}\right)$ with polarized beams at $\sqrt{s}=400$ and $500 \mathrm{GeV}$

- the forward-backward asymmetry $A_{\mathrm{FB}}$ at $\sqrt{s}=400$ and $500 \mathrm{GeV}$

- the Higgs boson mass, $m_{h}$

- the branching ratio $\mathcal{B}(b \rightarrow s \gamma)$

- the anomalous muon magnetic moment.

Compared to the previous scenarios, these observables are supplemented by the Higgs boson mass, $m_{h}$, calculated us- ing FeynHiggs 2.9.1 [56-59]. The estimated experimental precision at the LC for $m_{h}$, taken from Ref. [1], is adopted. We further assume the future theoretical uncertainty on the Higgs boson mass to be $1 \mathrm{GeV}$ [59]. As before, the remaining two observables, the branching ratio $\mathcal{B}(b \rightarrow s \gamma)$ and the anomalous muon magnetic moment are calculated using micrOmegas 2 . 4 . 1 [62, 63], and a projected experimental error on the anomalous muon magnetic moment of $3.4 \cdot 10^{-10}$ is employed [68], which we assume would dominate over the theoretical uncertainty. The input variables, together with errors, namely the assumed experimental precision of the prospective LC measurements and the theoretical uncertainties, are summarized in Table 6. Because the sneutrino is now directly accessible, we assume that its mass has been measured and it is therefore not included in the fit. On the other hand, due to the stronger dependence of the NLO cross-section and forward-backward asymmetry on $M_{A^{0}}$, this is now used as an additional fit parameter. We neglect the remaining theoretical uncertainty on the cross-sections, as it is found to be negligible in comparison to the experimental error.

This means that in scenario 3 , we fit to $M_{1}, M_{2}, \mu, \tan \beta$, $\cos \theta_{t}, m_{\tilde{t}_{1}}, m_{\tilde{t}_{2}}$ and $M_{A^{0}}$. The results of the fit are collected in Table 7. The parameters of the electroweak gauginohiggsino sector are determined with high precision. Due to a significant mixing in the stop sector, and the improvement in the fit quality due to the inclusion of the Higgs mass, we find that the fit is now also sensitive to the mass of the heavy stop. The accuracy is better than $20 \%$ for this particle even though it is far beyond the reach of the LC and also most likely of the LHC. In addition, in this scenario an upper limit on the mass of the heavy Higgs boson can be placed at $1000 \mathrm{GeV}$, at the $2 \sigma$ level. It is the particular sensitivity of the NLO corrections to $M_{A^{0}}$ which presents this unique opportunity to set such an upper bound. 
Table 6 Observables (masses in $\mathrm{GeV}$, cross sections in $\mathrm{fb}$ ) used as an input for the fit in S3, as in Table 2. The central values of the theoretical predictions $\mathcal{B}(b \rightarrow s \gamma)=2.7 \cdot 10^{-4}$, $\Delta\left(g_{\mu}-2\right) / 2=2.4 \cdot 10^{-9}$ and $m_{h}=125 \mathrm{GeV}$, calculated using state-of-the-art tools, are also included in the fit. See text for details of error estimation

\begin{tabular}{lclll}
\hline Observable & Tree value & Loop corr. & Error exp. & Error th. \\
\hline$m_{\tilde{\chi}_{1}^{ \pm}}$ & 139.3 & - & 0.1 & - \\
$m_{\tilde{\chi}_{2}^{ \pm}}$ & 266.2 & - & 0.5 & - \\
$m_{\tilde{\chi}_{1}^{0}}$ & 92.8 & - & 0.2 & - \\
$m_{\tilde{\chi}_{2}^{0}}$ & 148.5 & 2.4 & 0.5 & 0.5 \\
$m_{\tilde{\chi}_{3}^{0}}$ & 189.7 & -7.3 & 0.5 & 0.5 \\
$\sigma\left(\tilde{\chi}_{1}^{+} \tilde{\chi}_{1}^{-}\right)_{(-0.8,0.6)}^{400}$ & 709.7 & -85.1 & 4.5 & - \\
$\sigma\left(\tilde{\chi}_{1}^{+} \tilde{\chi}_{1}^{-}\right)_{(0.8,-0.6)}^{400}$ & 129.8 & 20.0 & 2.0 & - \\
$\sigma\left(\tilde{\chi}_{1}^{+} \tilde{\chi}_{1}^{-}\right)_{(-0.8,0.6)}^{500}$ & 560.0 & -70.1 & 4.5 & - \\
$\sigma\left(\tilde{\chi}_{1}^{+} \tilde{\chi}_{1}^{-}\right)_{(0.8,-0.6)}^{500}$ & 97.1 & 16.4 & 2.0 & - \\
$A_{\mathrm{FB}}^{400}(\%)$ & 24.7 & -2.8 & 1.4 & 0.1 \\
$A_{\mathrm{FB}}^{500}(\%)$ & 39.2 & -5.8 & 1.5 & 0.1 \\
\hline
\end{tabular}

Table 7 Fit results (in $\mathrm{GeV}$ with the exception of $\tan \beta$ and $\cos \theta_{t}$ ) for $\mathrm{S} 3$, including results for the masses of the heavier stop mass $\left(m_{\tilde{t}_{2}}\right)$ and the pseudoscalar Higgs boson $\left(M_{A^{0}}\right)$

\begin{tabular}{ll}
\hline Parameter & Fit result \\
\hline$M_{1}$ & $106 \pm 0.3( \pm 0.5)$ \\
$M_{2}$ & $212 \pm 0.5( \pm 1.0)$ \\
$\mu$ & $180 \pm 0.4( \pm 0.9)$ \\
$\tan \beta$ & $12 \pm 0.3( \pm 0.7)$ \\
$\cos \theta_{t}$ & $0.15_{-0.06}^{+0.08}\left({ }_{-0.09}^{+0.16}\right)$ \\
$m_{\tilde{t}_{1}}$ & $430_{-130}^{+200}\left({ }_{-400}^{+300}\right)$ \\
$m_{\tilde{t}_{2}}$ & $1520_{-300}^{+200}\left({ }_{-400}^{+300}\right)$ \\
$M_{A^{0}}$ & $<650(<1000)$ \\
\hline
\end{tabular}

\section{Conclusions}

The evidence for the Higgs boson and dark matter, when examined in the context of supersymmetry, suggests the possibility of a light $\mu$ and $M_{1}$. We have extended previous analyses, which fitted observables for chargino production at the LC to extract fundamental MSSM parameters, by incorporating NLO corrections. The loop corrections are calculated for all observables fitted, namely the polarized cross-sections and forward-backward asymmetry for chargino production as well as the $\tilde{\chi}_{1}^{ \pm}, \tilde{\chi}_{2}^{ \pm}$and $\tilde{\chi}_{1}^{0}, \tilde{\chi}_{2}^{0}, \tilde{\chi}_{3}^{0}$ masses, in an on-shell scheme which facilitates the extension to the complex case. We have fitted these observables for three complementary scenarios. We found that on including NLO corrections, when $M_{1}, M_{2}$ and $\mu$ are light they can be determined to percent-level accuracy, and $\tan \beta$ to $<5 \%$. Further we showed that obtaining masses of the charginos and neutralinos from the continuum as opposed to threshold scans would result in the uncertainty on the fundamental parameters almost doubling, reinforcing the importance of threshold scans for mass measurements. As a heavy $M_{2}$ is still a viable possibility, we also considered

$M_{2}=2000 \mathrm{GeV}$, and found that the sensitivity to $M_{2}$ is approximately $10 \%$. As the error on $\tan \beta$ is dependent on the degree of mixing in the chargino sector, here it increases to $\sim 20 \%$. Note that the inclusion of $\mathcal{B}(b \rightarrow s \gamma)$, as well as the use of masses determined via threshold scanning, in the fit was seen to improve the sensitivity to the stop sector. We finally considered a scenario compatible with the latest Higgs results. For this scenario we found that including $\mathcal{B}(b \rightarrow s \gamma), \Delta\left(g_{\mu}-2\right) / 2$ and $m_{h}$ in the fit, along with the significant mixing in the stop sector, helped to obtain an accuracy better than $20 \%$ on the mass of the heavy stop, even though this particle is far beyond the reach of the LC and also most likely of the LHC. We also included $M_{A^{0}}$ in the fit, and found that, due to the particular sensitivity of the NLO corrections to $M_{A^{0}}$, it would even be possible to place a $2 \sigma$ upper bound on this parameter of $1000 \mathrm{GeV}$. In summary, we have shown that incorporating NLO corrections is required for the precise determination of the fundamental parameters of the chargino and neutralino sector at the LC, and could further provide sensitivity to the parameters describing particles which contribute via loop corrections.

Acknowledgements The authors gratefully acknowledge support of the DFG through the grant SFB 676, "Particles, Strings, and the Early Universe", as well as the Helmholtz Alliance, "Physics at the Terascale". This work was also partially supported by the Polish National Science Centre under research grant DEC-2011/01/M/ST2/02466 and the MICINN, Spain, under contract FPA2010-17747; ConsoliderIngenio CPAN CSD2007- 00042. KR thanks as well the Comunidad de Madrid through Proyecto HEPHACOS S2009/ESP-1473 and the European Commission under contract PITN-GA-2009-237920.

\section{References}

1. J. Aguilar-Saavedra et al. (ECFA/DESY LC Physics Working Group Collaboration). hep-ph/0106315

2. K. Abe et al. (ACFA Linear Collider Working Group Collaboration). hep-ph/0109166 
3. T. Abe et al. (American Linear Collider Working Group Collaboration). hep-ex/0106056

4. J. Brau et al. (ILC Collaboration). arXiv:0712.1950

5. G. Aarons et al. (ILC Collaboration). arXiv:0709.1893

6. H. Goldberg, Phys. Rev. Lett. 50, 1419 (1983)

7. J.R. Ellis, J. Hagelin, D.V. Nanopoulos, K.A. Olive, M. Srednicki, Nucl. Phys. B 238, 453-476 (1984)

8. L.J. Hall, D. Pinner, J.T. Ruderman, J. High Energy Phys. 1204, 131 (2012). arXiv:1112.2703

9. F. Brummer, W. Buchmuller, J. High Energy Phys. 1107, 010 (2011). arXiv: 1105.0802

10. S. Chatrchyan et al. (CMS Collaboration), J. High Energy Phys. 1211, 147 (2012). arXiv: 1209.6620

11. G. Aad et al. (ATLAS Collaboration), Phys. Lett. B 718, 841-859 (2013). arXiv:1208.3144

12. P. Bechtle, T. Bringmann, K. Desch, H. Dreiner, M. Hamer et al., J. High Energy Phys. 1206, 098 (2012). arXiv:1204.4199

13. H. Baer et al. (ILC Collaboration), International Linear Collider Technical Design Report-Volume 1: Physics at the International Linear Collider (2012)

14. W. Oller, H. Eberl, W. Majerotto, Phys. Rev. D 71, 115002 (2005). hep-ph/0504109

15. T. Fritzsche, W. Hollik, Nucl. Phys. Proc. Suppl. 135, 102-106 (2004). hep-ph/0407095

16. W. Kilian, J. Reuter, T. Robens, Eur. Phys. J. C 48, 389-400 (2006). hep-ph/0607127

17. T. Robens, J. Kalinowski, K. Rolbiecki, W. Kilian, J. Reuter, Acta Phys. Pol. B 39, 1705-1714 (2008). arXiv:0803.4161

18. A. Bharucha, A. Fowler, G. Moortgat-Pick, G. Weiglein. arXiv: 1211.3134

19. T. Fritzsche, Ph.D. thesis, Universität Karlsruhe (2005)

20. A. Fowler, G. Weiglein, J. High Energy Phys. 1001, 108 (2010). arXiv:0909.5165

21. A. Fowler, Ph.D. thesis, Durham University (2010)

22. A. Chatterjee, M. Drees, S. Kulkarni, Q. Xu. arXiv:1107.5218

23. S. Heinemeyer, F. von der Pahlen, C. Schappacher, Eur. Phys. J. C 72, 1892 (2012). arXiv:1112.0760

24. A. Bharucha, S. Heinemeyer, F. von der Pahlen, C. Schappacher. arXiv: 1208.4106

25. H.E. Haber, G.L. Kane, Phys. Rep. 117, 75-263 (1985)

26. S. Choi, A. Djouadi, M. Guchait, J. Kalinowski, H. Song et al., Eur. Phys. J. C 14, 535-546 (2000). hep-ph/0002033

27. J. Kublbeck, M. Bohm, A. Denner, Comput. Phys. Commun. 60, $165-180$ (1990)

28. A. Denner, H. Eck, O. Hahn, J. Kublbeck, Nucl. Phys. B 387, 467-484 (1992)

29. J. Kublbeck, H. Eck, R. Mertig, Nucl. Phys. Proc. Suppl. 29A, 204-208 (1992)

30. T. Hahn, Comput. Phys. Commun. 140, 418-431 (2001). hep-ph/ 0012260

31. T. Hahn, C. Schappacher, Comput. Phys. Commun. 143, 54-68 (2002). hep-ph/0105349

32. T. Hahn, M. Perez-Victoria, Comput. Phys. Commun. 118, 153165 (1999). hep-ph/9807565

33. T. Hahn, Comput. Phys. Commun. 178, 217-221 (2008). hep-ph/0611273

34. T. Hahn, M. Rauch, Nucl. Phys. Proc. Suppl. 157, 236-240 (2006). hep-ph/0601248

35. W. Siegel, Phys. Lett. B 84, 193 (1979)

36. W. Siegel, Phys. Lett. B 94, 37 (1980)
37. D. Stockinger, J. High Energy Phys. 0503, 076 (2005). hep-ph/ 0503129

38. F. del Aguila, A. Culatti, R. Munoz Tapia, M. Perez-Victoria, Nucl. Phys. B 537, 561-585 (1999). hep-ph/9806451

39. A. Lahanas, K. Tamvakis, N. Tracas, Phys. Lett. B 324, 387-396 (1994). hep-ph/9312251

40. D. Pierce, A. Papadopoulos, Phys. Rev. D 50, 565-570 (1994). hep-ph/9312248

41. D. Pierce, A. Papadopoulos, Nucl. Phys. B 430, 278-294 (1994). hep-ph/9403240

42. H. Eberl, M. Kincel, W. Majerotto, Y. Yamada, Phys. Rev. D 64, 115013 (2001). hep-ph/0104109

43. T. Fritzsche, W. Hollik, Eur. Phys. J. C 24, 619-629 (2002). hep-ph/0203159

44. W. Oller, H. Eberl, W. Majerotto, C. Weber, Eur. Phys. J. C 29, 563-572 (2003). hep-ph/0304006

45. M. Drees, W. Hollik, Q. Xu, J. High Energy Phys. 0702, 032 (2007). hep-ph/0610267

46. R. Schofbeck, H. Eberl, Phys. Lett. B 649, 67-72 (2007). hep-ph/0612276

47. R. Schofbeck, H. Eberl, Eur. Phys. J. C 53, 621-626 (2008). arXiv:0706.0781

48. K. Rolbiecki, J. Kalinowski, Phys. Rev. D 76, 115006 (2007). arXiv:0709.2994

49. H. Eberl, T. Gajdosik, W. Majerotto, B. Schrausser, Phys. Lett. B 618, 171-181 (2005). hep-ph/0502112

50. P. Osland, A. Vereshagin, Phys. Rev. D 76, 036001 (2007). arXiv:0704.2165

51. K. Desch, J. Kalinowski, G. Moortgat-Pick, K. Rolbiecki, W. Stirling, J. High Energy Phys. 0612, 007 (2006). hep-ph/0607104

52. F. James, M. Roos, Comput. Phys. Commun. 10, 343-367 (1975)

53. F. James, CERN Program Library Long Writeup D506 (1994)

54. G. Aad et al. (ATLAS Collaboration). arXiv:1208.0949

55. S. Chatrchyan et al. (CMS Collaboration). arXiv:1207.1798

56. S. Heinemeyer, W. Hollik, G. Weiglein, Comput. Phys. Commun. 124, 76-89 (2000). hep-ph/9812320

57. S. Heinemeyer, W. Hollik, G. Weiglein, Eur. Phys. J. C 9, 343-366 (1999). hep-ph/9812472

58. G. Degrassi, S. Heinemeyer, W. Hollik, P. Slavich, G. Weiglein, Eur. Phys. J. C 28, 133-143 (2003). hep-ph/0212020

59. M. Frank, T. Hahn, S. Heinemeyer, W. Hollik, H. Rzehak et al., J. High Energy Phys. 0702, 047 (2007). hep-ph/0611326

60. G. Aad et al. (ATLAS Collaboration), Phys. Lett. B 716, 1-29 (2012). arXiv:1207.7214

61. S. Chatrchyan et al. (CMS Collaboration), Phys. Lett. B 716, 30 61 (2012). arXiv:1207.7235

62. G. Belanger, F. Boudjema, A. Pukhov, A. Semenov, Comput. Phys. Commun. 176, 367-382 (2007). hep-ph/0607059

63. G. Belanger, F. Boudjema, P. Brun, A. Pukhov, S. RosierLees et al., Comput. Phys. Commun. 182, 842-856 (2011). arXiv: 1004.1092

64. E. Komatsu et al. (WMAP Collaboration), Astrophys. J. Suppl. 192, 18 (2011). arXiv: 1001.4538

65. R. Aaij et al. (LHCb Collaboration), Phys. Rev. Lett. 110, 021801 (2013). arXiv:1211.2674

66. B. O'Leary et al. (SuperB Collaboration). arXiv:1008.1541

67. G. Weiglein et al. (LHC/LC Study Group Collaboration), Phys. Rep. 426, 47-358 (2006). hep-ph/0410364

68. R. Carey, K. Lynch, J. Miller, B. Roberts, W. Morse et al., Preprint, FERMILAB-PROPOSAL-0989, 2009 